\title{
ANÁLISE REFLEXIVA ACERCA DAS CONTRIBUIÇÕES DA TECNOLOGIA DA INFORMAÇÃO VERDE PARA A SUSTENTABILIDADE CORPORATIVA
}

\author{
http://dx.doi.org/10.21527/2237-6453.2021.56.11205
}

Recebido em: 25/7/2020

Aceito em: 12/5/2021

Vanessa Theis, ${ }^{1}$ Dusan Schreiber ${ }^{2}$

\begin{abstract}
RESUMO
Conduzido por meio de uma revisão sistemática da literatura, este artigo objetiva situar o modelo da Tecnologia da Informação Verde (TI Verde) no contexto teórico da Sustentabilidade Corporativa. Tendo em vista que as práticas de TI Verde são utilizadas com o propósito de reduzir os impactos ambientais causados pelas atividades de TI, uma análise reflexiva quanto ao uso de medidas associadas à TI Verde possibilita identificar o conjunto de atividades que precisam ser realizadas para as empresas tornarem-se ambientalmente corretas, a fim de introduzirem processos de negócios ambientalmente favoráveis nas organizações e produtos ambientalmente corretos para o mercado. A pesquisa originou-se a partir da percepção da complexidade que caracteriza a referida temática, decorrente da evolução tecnológica no âmbito de Tecnologia de Informação, que contribui e reforça a necessidade de realização de estudos de caráter reflexivo que possam facultar a concepção de modelos de gestão de TI eficazes e de menor custo para a sua operacionalização nas organizações.
\end{abstract}

Palavras-chave: TI verde; Sustentabilidade, triple botton line.

\section{REFLECTIVE ANALYSIS ON GREEN INFORMATION TECHNOLOGY CONTRIBUTIONS TO CORPORATE SUSTAINABILITY}

\section{ABSTRACT}

Conducted through a systematic literature review, this article aims to situate the model of Green Information Technology in the theoretical context of Corporate Sustainability. Considering that Green IT practices are used to reduce the environmental impacts caused by IT activities, a reflexive analysis regarding the use of measures associated to Green IT allows identifying the set of activities that need to be performed for the environmentally sound business processes in organizations and products that are environmentally sound for the market. The research originated from the perception of the complexity that characterizes the aforementioned thematic, due to the technological evolution in the field of information technology, which contributes and reinforces the need to carry out reflective studies that may allow the design of management models cost effective IT solutions for their operation in organizations.

Keywords: green IT; Sustainability; triple bottom line.

\footnotetext{
${ }^{1}$ Autora correspondente. Universidade Feevale. RS-239, 2755 - Vila Nova. Novo Hamburgo/RS, Brasil. CEP 93525-075. http://lattes. cnpq.br/4178432531987990. https://orcid.org/0000-0002-5100-7574. nessa.theis@gmail.com

${ }^{2}$ Universidade Feevale. Novo Hamburgo/RS, Brasil. http://lattes.cnpq.br/5088882022022801. dusan@feevale.br
} 


\section{INTRODUÇÃO}

Inserida de maneira central na sociedade, o desenvolvimento da Tecnologia de Informação (TI) gerou a configuração de novos cenários competitivos, pois possibilitou que as organizações avançassem no processo de oferta de produtos e serviços. Em contrapartida, a TI contribuiu, de forma significativa, para o aumento dos problemas ambientais com os quais a sociedade atual se depara, principalmente devido ao elevado consumo de energia elétrica, quantidade de insumos não renováveis utilizados na produção de computadores e periféricos, além do descarte inadequado de equipamentos obsoletos.

Neste contexto, surge o conceito da Tecnologia da Informação Verde (TI Verde) caracterizado pela revisão das estruturas de $\mathrm{TI}$, a fim de incorporar o aspecto ambiental da sustentabilidade, no que diz respeito à eficiência energética, planejamento e investimento em uma infraestrutura tecnológica que atenda às necessidades de hoje, conservando os recursos necessários para as gerações futuras (DENG; Jl; WANG, 2017). Ademais, dado o contexto, cada vez mais competitivo, da economia contemporânea, decorrente da evolução tecnológica, por meio da reformulação do modelo de negócios, é possível que as empresas integrem mais facilmente a sustentabilidade em seus negócios, a fim de maximizar os benefícios sociais e ambientais, em vez de contemplar apenas o ganho econômico.

No cenário da pesquisa brasileira, a busca pelas palavras-chave "TI Verde", "Tecnologia da Informação Verde", "Green IT" e "Green Information Technology" na plataforma de busca SClelo, encontrou apenas 5 artigos, na data de 10 de fevereiro de 2021. Ressalta-se que a busca por palavras foi realizada no campo do título, sem nenhum filtro em relação ao ano de publicação, conforme disponibilidade daquela base de dados. Em busca no portal SPELL, também em 10 de fevereiro de 2021, utilizando as palavras-chave citadas e sem delimitação quando no momento da publicação, foram encontrados 12 artigos, além dos já encontrados no portal SClelo.

Com base na constatação de que poucos estudos sobre o tema TI Verde foram realizados no Brasil, este artigo, conduzido por meio de uma revisão sistemática da literatura, objetiva situar o modelo da Tecnologia da Informação Verde no contexto teórico da Sustentabilidade Corporativa. Após esta introdução, o artigo apresenta os procedimentos metodológicos utilizados na execução desta pesquisa, seguida da revisão das bases conceituais sobre Sustentabilidade Corporativa e TI Verde, destacando-se que os aspectos estruturantes, subjacentes à construção do modelo pretendido, são apresentados na última seção do trabalho. Por fim, são descritas as considerações em relação aos artigos analisados, as contribuições do estudo, as limitações da pesquisa e sugestões para investigações futuras.

\section{PROCEDIMENTOS METODOLÓGICOS}

Em alinhamento com o objetivo da pesquisa de situar o modelo da TI Verde no contexto teórico da Sustentabilidade Corporativa, realizou-se um estudo de caráter qualitativo, visto que se utiliza da interpretação subjetiva de informações textuais. Como procedimento de pesquisa realizou-se uma revisão sistemática da literatura, utilizando-se da pesquisa bibliográfica como técnica de coleta de dados. Os autores Wolfswinkel, Furtmueller e Wilderom (2013) destacam que a revisão sistemática procura fornecer uma avaliação rigorosa do progresso teórico e dos estudos empíricos. 
Para Kitchenham et al. (2010), a revisão sistemática da literatura é uma forma de agregar conhecimento e reunir saberes sobre um tema, sendo composta por três fases: a) planejamento da revisão, quando identifica-se a sua necessidade; b) realizar a revisão, quando identifica-se a investigação, a seleção dos estudos com avaliação de estudos relevantes, extração dos dados para análise e síntese dos resultados e c) relato da revisão. Ridley (2012) ressalta que a revisão sistemática da literatura baseia-se em um processo estruturado e transparente, de modo a ser replicável, com isso permitindo que as decisões e conclusões do revisor possam ser verificadas.

Neste sentido, a pesquisa foi realizada observando-se a sistemática sugerida por Fink (2014), que compreende as seguintes atividades: a) escolher o tema: TI Verde e Sustentabilidade Corporativa; b) selecionar as bases de dados; c) escolher os termos de pesquisa; d) aplicar os critérios práticos de seleção; e) aplicar os critérios de seleção metodológica. Embora as atividades empregadas tenham sido as mesmas para as duas temáticas do estudo, cada uma delas possui características próprias.

Com base nas revisões de literatura realizadas por Jenkin, Webster e McShane (2011), Malhotra, Melville e Watson (2013), Asadi, Hussin e Dahlan (2017) e García - Berná (2019) verificou-se que o termo "TI Verde" foi utilizado pela primeira vez em 2007, justificando assim a data inicial para a seleção da bibliografia sobre o referido tema. Deve-se observar que Jenkin, Webster e McShane (2011) desenvolveram uma pesquisa com foco na literatura de gestão, psicologia ambiental e marketing social, ou seja, utilizaram pesquisas ambientais sobre indivíduos e organizações.

Malhotra, Melville e Watson (2013) concentraram suas pesquisas em estudos que apresentam soluções para mitigar as mudanças climáticas, excluindo aqueles que apenas conceituam e analisam o tema TI Verde. Finalmente, Asadi, Hussin e Dahlan (2017) analisaram 131 artigos com o objetivo de obter um melhor entendimento da área de TI Verde, apresentando um roteiro para orientar estudos futuros e destacar as práticas de efetivação de TI Verde.

Ressalta-se que os autores supracitados realizaram sua revisão bibliográfica focando os portais de periódicos e congressos específicos. No que diz respeito à metodologia utilizada neste estudo, e que difere de trabalhos anteriores, é que a pesquisa bibliográfica foi realizada em bases de dados, a fim de expandir a pesquisa para áreas interdisciplinares, visto que, segundo Webster e Watson (2002), a Tl é uma área interdisciplinar que se encaixa em outras disciplinas. Outra diferença, no caso desta pesquisa, é que o maior número de artigos provém de periódicos e não de congressos.

Para a pesquisa bibliográfica sobre a temática de TI Verde foram utilizadas as seguintes bases de dados: ABI/INFORM Global, AIS Electronic Library, JSTOR Archival Journals, Science Direct, Scopus e Wiley Online Library. As consultas foram realizadas entre os dias 18 e 22 de junho de 2020, utilizando como palavras-chave: "Green IT", "Green IS", "Green Computer", "Green ICT", "Green Information Technology" e "Green Computing". Os termos foram propostos entre aspas duplas, de forma que os resultados da busca apresentassem apenas artigos dentro do contexto semântico desejado. Em relação às áreas de estudo, os filtros utilizados foram: gestão de tecnologia da informação, informática, administração de empresas, gestão, sustentabilidade, desenvolvimento sustentável e meio ambiente.

Os autores Webster e Watson (2002), no entanto, sugerem a consulta de artigos publicados em anais de eventos científicos, em razão de sua contemporaneidade, que se caracteriza 
pelo ritmo acelerado de inovação tecnológica. Sendo assim, selecionaram-se artigos divulgados em conferências que são suportadas pela Associação de Sistemas de Informação (AIS). Desse modo, foram revisados os anais dos seguintes eventos: Hawaii International Conference on System Sciences (HICSS), Australian Conference on Information Systems (Acis), International Conference on Information Systems (Icis), American Conference on Information Systems (Amcis), Pacific Asia Conference on Information Systems (Pacis) e European Conference on Information Systems (Ecis).

Com relação aos critérios adotados para seleção de bibliografia, também denominados por Fink (2014) como critérios de inclusão e exclusão, estabeleceu-se: o ano de publicação, somente artigos no idioma inglês e a abordagem sobre sustentabilidade e Tecnologia da Informação no ambiente organizacional. Os artigos foram selecionados por meio da leitura de seus títulos, resumos, palavras-chave e objetivos, a fim de identificar se atendiam aos critérios supramencionados. Este processo foi realizado até que as bibliografias começassem a se repetir, configurando o princípio de saturação apresentado por Eisenhardt (1989). A saturação teórica é o ponto em que o aprendizado incremental é mínimo, ou, no caso deste estudo, quando os artigos advindos dos filtros de busca começassem a se repetir, não trazendo nenhum estudo novo.

Como resultado desta estratégia de pesquisa foram selecionados 171 artigos, que forneceram uma visão geral do tema abordado ou focaram um aspecto particular da TI Verde. A próxima etapa diz respeito à aplicação dos critérios de seleção metodológica, que, de acordo com Fink (2014), consiste em identificar os estudos com melhor qualidade. Para tal fim utilizou-se a técnica de rastreio de citações descrita por Webster e Watson (2002), uma vez que este é um indicador do impacto e da qualidade dos estudos. Considerou-se para análise inicial apenas os artigos mais citados pelo CrossRef (citados mais de 20 vezes), atingindo-se um resultado de 33 artigos em periódicos e 11 trabalhos publicados em anais de eventos científicos.

Observou-se, no entanto, que 31 dos artigos selecionados foram publicados em revistas científicas, entre os anos de 2020 e 2016; logo, não se espera que no curto prazo possam alcançar um número elevado de citações. Com o propósito de considerá-los na pesquisa, a fim de manter os dados o mais atualizado possível, estipulou-se como critério de seleção metodológica o fator de impacto mínimo da revista em 3. Os autores Sims e McGhee (2003) sinalizam que o fator de impacto dos periódicos é empregado para avaliar a importância de um dado periódico em sua área, não existindo um número mínimo de fator de impacto. Para definir se uma revista possui um bom fator de impacto os referidos autores aconselham compará-la com outras revistas da mesma categoria de assunto.

Nesta perspectiva, a partir das revisões teóricas sobre TI Verde realizadas por Jenkin, Webster e Mcshane (2011), Malhotra, Melville e Watson (2013) e Asadi et al. (2017) constatou-se que a seleção de artigos para análise ocorreu em periódicos cujo fator de impacto aproximou-se, ou foi superior, a 3, sendo este o fator de corte utilizado neste estudo. Desta forma, foram incluídos mais 10 estudos no escopo final da pesquisa. Sendo assim, a análise final compreende a revisão de 54 artigos sobre TI Verde.

Para a pesquisa bibliográfica sobre sustentabilidade, utilizaram-se as seguintes palavras-chave: a) Sustainability; b) Concept of sustainability; e c) Concept of sustainable development, nas bases de dados da Science Direct, Scopus e Wiley Online Library, no mês de março de 2018. Estes termos foram inseridos na aba de busca avançada, com aspas duplas e separados por um 
sinal de ponto e vírgula, com a busca sendo configurada para localizar os termos nos títulos, resumos e palavras-chave e considerando todo o período de abrangência de cada base de dados.

Tendo em vista que as plataformas da Science Direct e Scopus possibilitam o ordenamento dos artigos quanto a sua relevância e ao número de vezes que foram citados, foi possível identificar a contemporaneidade da temática e os autores mais referenciados. Para cada pesquisa realizada, com os termos em conjunto ou separados, procedeu-se à leitura dos resumos considerando-se os seguintes critérios de inclusão: a) estar disponível na base de dados; b) ser um estudo teórico ou empírico da área de conhecimento da administração; c) estar escrito no idioma inglês; d) publicado em periódico que utiliza o processo double blind review. Após aplicação destes critérios selecionaram-se 151 artigos, distribuídos em: Scopus (86), Wiley (33) e Science Direct (32).

Realizou-se análise textual dos artigos, tanto de Sustentabilidade como de TI Verde, a fim de sintetizar as ideias e concepções para se ter uma profunda compreensão das informações textuais, e, desta forma, identificar os principais conceitos utilizados pelos autores. Cumpre destacar que esta pesquisa bibliográfica não pretendeu esgotar a revisão da literatura sobre Sustentabilidade e TI Verde, e, tampouco abordar toda a temática, mas coletar informações suficientes para atender ao objetivo da pesquisa e verificar o ineditismo da temática.

\section{FUNDAMENTAÇÃO TEÓRICA}

Com o intuito de contemplar a proposta da pesquisa, nesta seção discorre-se sobre os conceitos seminais que contribuíram para a edificação das bases teóricas acerca do conceito TI Verde e Sustentabilidade Corporativa.

\section{Tecnologia da Informação Verde}

Em termos conceituais Elliot (2007) sinaliza que a TI Verde é considerada o ciclo de vida completo das tecnologias de informação e de comunicação, envolvendo processos ambientalmente corretos de projetos de design, produção, operação e de eliminação (ELLIOT, 2007). Destarte, evidencia-se que o conceito de TI Verde vem sendo utilizado como um termo genérico para medidas e atividades relacionadas à $\mathrm{TI}$, que buscam contribuir com os aspectos da sustentabilidade ambiental e da responsabilidade social das empresas (CHEN; BOUDREAU; WATSON, 2008) e não apenas com as questões de consumo consciente (FUCHS, 2008).

Isto ocorre devido à TI Verde incorporar o aspecto ambiental da sustentabilidade, no que diz respeito à eficiência energética, planejar e investir em uma infraestrutura tecnológica que atenda às necessidades de hoje, conservando os recursos necessários para as gerações futuras, além de economizar dinheiro (POLLACK, 2008). Ressalta-se que em termos conceituais, Molla et al. (2009) propõem que a TI Verde é a capacidade de uma organização em aplicar sistematicamente critérios de sustentabilidade ambiental, tais como prevenção da poluição, administração de produtos e uso de tecnologias limpas, nos processos de produção, abastecimento, uso e disposição da infraestrutura técnica de TI.

Neste encadeamento temático, Sarkar e Young (2009) destacam que as organizações estão sendo mais exigidas e pressionadas por reguladores governamentais e órgãos de vigilância ambiental, para alinhar seus negócios com as práticas de sustentabilidade ambiental. Kuo e Dick 
(2010) complementam que as pressões competitivas do mercado também exercem influência sobre a decisão de adotar práticas ambientalmente sustentáveis, e acreditam que o principal fator de diferencial competitivo, neste cenário, é a capacidade de a organização se adaptar à nova realidade e às novas tecnologias.

Mithas, Khuntia e Roy (2010) defendem que a consolidação de TI Verde está positivamente associada a maiores reduções no consumo de energia dos equipamentos de $\mathrm{Tl}$ e, consequentemente, maior impacto nos lucros. Cumpre destacar que Watson, Boudreau e Chen (2010) entendem que buscar a sustentabilidade não significa abandonar o pensamento econômico. Isto porque a economia é direcionada para o problema de alocar recursos escassos, e recursos como as energias livres de emissão de gases e componentes eletroeletrônicos são particularmente escassos. Logo, o gerenciamento inteligente da tecnologia é uma alternativa às empresas para reduzir os danos causados ao meio ambiente, melhorar a efetividade do consumo de energia elétrica e reduzir os custos operacionais do negócio (MELVILLE, 2010).

Na visão de Corbett (2010), a TI Verde é definida como o conjunto de tecnologias de informação e comunicação e sistemas de informação que são, direta ou indiretamente, usados para reduzir os impactos ambientais nocivos das atividades humanas. Para as organizações, as iniciativas da TI Verde podem estar relacionadas com a aquisição de hardwares eficientes em energia, gerenciamento de energia do usuário final, reciclagem e redução de resíduos eletrônicos, práticas de teleconferência, virtualização de informações, design e gerenciamento dos centros de dados, sistemas de gerenciamento de carbono e relatórios ambientais. Os autores Dick e Burns (2011) apontam que a economia no consumo de papel é uma das práticas de TI Verde mais difundidas entre as organizações.

Unhelkar (2011) ressalta que os gestores precisam melhorar a eficiência energética da organização por meio de estratégias de gestão inovadoras, sendo que estas estratégias precisam aliar custos operacionais com as emissões de carbono despendidas pelos datacenters. Referente a infraestrutura de $\mathrm{TI}$, o autor cita o uso de armazenamento de dados virtualmente, a fim de reduzir o consumo enérgico. Por meio da Internet, é possível criar ambientes virtuais de trabaIho e os benefícios são mensurados, não apenas na diminuição do consumo de energia, mas na redução dos gastos com depreciação e manutenção (UNHELKAR, 2011; BUTLER, 2011).

Face ao exposto, Loos et al. (2011) salientam que o mundo enfrenta uma crise ecológica sob a forma de aquecimento global, resultante da liberação de $\mathrm{CO}_{2}$. No entanto, defendem aumento do investimento em pesquisa sobre como melhorar a eficiência energética da $\mathrm{TI}, \mathrm{com}$ foco especial na redução do consumo de energia por meio do desenvolvimento de soluções práticas que alavanquem o poder de transformação dos sistemas de informação. Em detrimento deste cenário, Molla e Abareshi (2011) constataram, em sua pesquisa, que os motivos de ecoeficiência e a ecoeficácia influenciam a adoção de tecnologias que melhoram a eficiência energética da infraestrutura de TI e subsequente redução da poluição.

Em termos práticos, o fato de a ecoeficiência e a ecoeficácia serem determinantes importantes da adoção da TI Verde, implica que há ganhos tangíveis positivos em termos de custos e proventos intangíveis como a liderança e a divulgação do pensamento ambiental associados à TI Verde. Molla e Abareshi (2012) complementam que, embora algumas das práticas exijam elevados investimentos, boa parte delas pode ser adotada sem que a saúde financeira da em- 
presa seja comprometida, dependendo apenas do esforço e vontade dos usuários e do apoio e direcionamento dos gestores.

Ressalta-se que a ecoeficiência está alinhada com a eficiência organizacional e a rentabilidade. Reduzir o consumo de energia e o uso de recursos é claramente uma maneira de reduzir os custos e melhorar as receitas. Para alcançar uma organização sustentável ambientalmente, contudo, os gestores precisam pensar e atuar de forma proativa, buscando soluções além das práticas convencionais de TI Verde, não se limitando apenas em agir de forma ambientalmente correta em prol de benefícios financeiros ou para cumprimento das legislações ambientais. Neste sentido, Chen et al. (2011) sinalizam que, a ecoefetividade tem por objetivo solucionar a questão ambiental, não importando a viabilidade financeira. Logo, a TI pode transformar uma empresa em direção à sustentabilidade ecológica, ao inserir inovações que mudam a lógica de comercialização ou uso de produtos ou serviços.

Centrando-se nos fatores motivacionais internos e externos à empresa, Molla e Abareshi (2011) inferiram que, quando os mecanismos regulatórios e de mercado não são suficientemente fortes, ou pouco claros, para encorajar a adoção da TI Verde, os gerentes que visam além da conformidade regulamentar provavelmente se beneficiarão ao investir na TI Verde. A percepção e a atitude dos funcionários, contudo, são tão importantes, se não mais, como o compromisso da administração e a alocação de recursos na transformação para tornar uma operação de TI sustentável.

Além do mais, Chen, Chang e Wu (2012) afirmam que os consumidores estão mais atentos às empresas que tentam inserir valores ambientalistas, mediante o uso de greenwash, a fim de criar uma imagem inexistente acerca do grau de responsabilidade ambiental diante dos stakeholders e clientes. Em detrimento desta realidade, Cai, Chen e Bose (2013) elucidam que o efeito significativo da TI Verde vai além da redução de custos. O uso adequado dos princípios da TI Verde para a sustentabilidade ambiental reforça a diferenciação no mercado, uma vez que melhora produtos e processos, aumentando a participação no mercado, ou possibilitando entrar em novos e mais lucrativos mercados. Sumariamente, com o uso adequado de sistemas e equipamentos relacionados à $\mathrm{Tl}$, uma empresa pode aumentar sua competitividade e ser ambientalmente amigável.

Com relação ao comportamento dos indivíduos para adoção das práticas da TI Verde, Mishra, Akman e Mishra (2014) observaram que os profissionais que identificam o valor agregado que a TI Verde pode propiciar, consideram as questões de sustentabilidade ambiental durante a compra de hardwares e softwares. Neste contexto, Koo, Chung e Nam (2015) destacam que a utilidade percebida das práticas TI Verde tem efeitos positivos sobre o comportamento e na intenção de continuidade de sua aplicação e utilização nos processos decisórios. Complementarmente, as organizações que almejam êxito na continuidade da TI Verde nos processos organizacionais precisam desenvolver técnicas de persuasão que modifiquem as intenções dos colaboradores, com atenção às atitudes, às normas subjetivas e ao controle percebido sobre o comportamento.

\section{Sustentabilidade Corporativa}

O Relatório Brundtland (1987) estabeleceu o conceito do desenvolvimento sustentável, aliando o crescimento econômico com o desenvolvimento social. No relatório, o conceito de desenvolvimento sustentável sugere a adoção de um modelo de desenvolvimento que possa 
atender às necessidades do presente sem comprometer a possibilidade das gerações futuras de suprir suas próprias necessidades. Em suma, o desenvolvimento sustentável prevê o uso consciente dos recursos naturais, tendo por objetivo o crescimento dos países, combate à pobreza e garantindo atendimento às necessidades humanas básicas (ONU, 1987).

Destarte, evidencia-se que a relação entre o desenvolvimento econômico e o meio ambiente surge com o esgotamento dos recursos naturais, a partir da expansão da atividade econômica. Estas questões intensificaram, inclusive, a atenção de consumidores, organizações ecológicas e entidades reguladoras sobre o impacto ambiental negativo das empresas: emissões de gases geradores do efeito estufa, contaminação por resíduos e uso ineficiente de água e energia (ELKINGTON; BURKE, 1987).

No ano em que o Relatório Brundtland foi publicado, o sociólogo inglês John Elkington, cunhou o termo sustentabilidade por meio de sua obra The Green Capitalists. Para o referido autor, sustentabilidade é, na realidade, um modelo de gestão de negócios que visa ao lucro para os acionistas, envolvendo, a um só tempo, o desenvolvimento econômico, a promoção social e a proteção dos recursos naturais do planeta. Este modelo de gestão foi denominado, pelo autor, de Triple Bottom Line e compreende os resultados de uma organização, medidos em termos sociais, ambientais e econômicos (ELKINGTON; BURKE, 1987).

Aprofundando o debate do conceito Triple Bottom Line, Dyllick e Hockerts (2002) evidenciam que a sustentabilidade corporativa pode ser definida como o atendimento das necessidades de todos os stakeholders, sem comprometer a capacidade de satisfazer às necessidades das futuras partes interessadas. Para tal finalidade as empresas precisam manter, e ampliar, sua base de capital econômico, social e ambiental, contribuindo ativamente para a sustentabilidade no domínio político. Destaca-se que a contribuição mais importante do conceito de sustentabilidade, na teoria de gestão ortodoxa, reside na sua percepção de que a sustentabilidade econômica, por si só, não é condição suficiente para a sustentabilidade geral de uma empresa.

Impulsionadas pelo mercado de ações, no entanto, as empresas tendem a enfatizar os ganhos de curto prazo, concentrando-se mais nos resultados imediatos do que nas estratégias de receitas de longo prazo. Outrossim, Dyllick e Hockerts (2002) concluem que focar apenas na sustentabilidade econômica pode ter sucesso no curto prazo, contudo, no longo prazo, a sustentabilidade exige que as três dimensões sejam satisfeitas simultaneamente. Desse modo, as empresas precisam gerenciar não apenas seu capital econômico, mas também seu capital ecológico e social.

Não obstante, Hart e Milstein (2003) explicitam que conciliar a sustentabilidade ambiental com o objetivo de aumentar o valor para o acionista, é um grande desafio, devido aos modelos de negócios obsoletos. Para alguns gestores, a sustentabilidade é uma obrigação moral, para outros uma exigência legal e, na maioria das vezes, ela é percebida como um custo de se fazer negócios, isto é, um mal necessário para manter a legitimidade organizacional, assegurando o direito de operar. A questão central é que alguns gestores acreditam que a criação de um mundo mais sustentável exigirá que as empresas sacrifiquem lucros e valor para os acionistas, em favor do patrimônio ambiental público.

Sumariamente, os desafios globais associados à sustentabilidade, observados sob a ótica dos negócios, podem ajudar a identificar estratégias e práticas que contribuem para um mundo mais sustentável e, simultaneamente, impulsionar o valor para os acionistas, representando, 
assim, a fonte de criação de valor sustentável para a empresa. Ademais, Hart e Milstein (2003) esclarecem que as oportunidades de se criar valor sustentável são enormes, mas ainda pouco exploradas, pois, embora possuam uma estrutura simples, as estratégias de execução tendem a ser complexas e desafiadoras, sugerindo que as iniciativas devem ser avaliadas usando um conjunto separado de critérios e métricas, uma vez que dificilmente atingirão as metas de receita e lucratividade de curto prazo, associadas ao que foi projetado para expandir os negócios existentes.

Hubbard (2009) corrobora esta percepção, ao afirmar que medir o desempenho organizacional com relação às práticas de sustentabilidade não é uma tarefa fácil, devido à inexistência de um consenso com relação ao conceito de sustentabilidade e, tampouco, quanto ao escopo das opções de medição. Geralmente, as medidas ambientais da Triple Bottom Line fazem referência à quantidade de recursos naturais que uma empresa utiliza em suas operações, tais como energia, terra e água, bem como aos subprodutos que suas atividades criam, como resíduos, emissões atmosféricas e poluentes químicos. O desempenho social evidencia o impacto que uma empresa e seus fornecedores geram nas comunidades em que trabalha.

Baumgartner e Ebner (2010) salientam que a dimensão econômica da sustentabilidade corporativa é frequentemente discutida de forma genérica. Uma análise mais abrangente compreende a relação entre a sustentabilidade corporativa, estratégias competitivas corporativas e aspectos de sustentabilidade. Nesse sentido, uma organização que tem sua estratégia de negócios alinhada com os princípios da sustentabilidade, possui maior credibilidade, visto que a estratégia de sustentabilidade é favorável e essencial para a estratégia competitiva. Além disso, Dangelico e Pujari (2010) entendem que esta relação resulta na diferenciação de produtos e aumento de vantagem competitiva no mercado.

Novas expectativas sociais também tornam imperativo para as empresas entender como integrar questões de sustentabilidade às estratégias de negócios, e, por conseguinte, em seus produtos. Nessa perspectiva, Dangelico e Pujari (2010) esclarecem que vários benefícios podem surgir da integração de questões de sustentabilidade ambiental ao desenvolvimento de produtos e operações comerciais: maior eficiência no uso de recursos, retorno sobre o investimento, aumento de vendas, desenvolvimento de novos mercados e melhor imagem corporativa. Os autores também expõem que as regulamentações ambientais não apenas representam restrições ou conformidades regulatórias, mas oferecem oportunidades para a minimização de riscos, preservação de receitas e reputação ou para a criação de novos negócios.

Sheth, Sethia e Srinivas (2011) defendem que uma agenda de sustentabilidade corporativa pode ser perseguida, com eficácia significativamente maior, quando o elemento central é a sustentabilidade centrada no cliente. $O$ termo alavanca a reciprocidade entre os negócios e os consumidores, ajudando a tornar a sustentabilidade uma parte integrante da estratégia e das operações de negócios. Propõe-se conceituar a sustentabilidade centrada no cliente como uma métrica de desempenho, com base em resultados financeiros advindos de ações comerciais de sustentabilidade direcionadas ao cliente. Como o marketing é a principal função de negócios voltada para o cliente, as suas ações constituem os impulsionadores de negócios mais relevantes.

Devido ao enfoque crescente nos esforços de sustentabilidade, o marketing eleva seu foco de gerenciamento para um conjunto mais amplo de problemas de mercado. Organizações que enfatizam a sustentabilidade, com foco no mercado, criam idiossincrasias exclusivas que afetam positivamente sua posição no mercado, pois possibilitam a segmentação de mercado 
e agregação de valor aos clientes. Sem este foco, nas demais partes interessadas, a Responsabilidade Social Corporativa não pode ser elevada ao nível de recurso estratégico e, tampouco, vinculada à vantagem competitiva e ao desempenho organizacional (HULT, 2011).Neste sentido, Crittenden et al. (2011) complementam que a referida abordagem possibilita a diferenciação de mercado por meio de recursos intangíveis.

Aguinis e Glavas (2012) destacam que as empresas se envolvem com práticas de sustentabilidade devido aos resultados financeiros esperados, por razões normativas atreladas aos valores da companhia, melhores práticas de gerenciamento, qualidade do produto, eficiência operacional, atratividade para os investidores e maior diversidade demográfica. Forças institucionais, incluindo regulamentações, padrões e certificações, também afetam a extensão e os tipos de ações e políticas de sustentabilidade que as empresas decidem colocar em prática. No que se refere aos efeitos da relação iniciativas e resultados, é possível constatar que os resultados são mais efetivos quando as partes interessadas têm mais poder e legitimidade e na presença de maior regulamentação.

Ampliando o debate acerca da definição de sustentabilidade, Baumgartner (2014) embasa-o no conceito de ecoeficiência, em que economia e ecologia são combinadas para o fornecimento de bens e serviços sustentáveis. O objetivo é que a ética, equidade e justiça sejam o foco central da interpretação normativa do desenvolvimento sustentável, visto que para atender às necessidades das gerações presentes e futuras estes três princípios devem ser contemplados. Os autores destacam que a igualdade de direitos das pessoas aos recursos ambientais e a responsabilidade social das empresas para as futuras gerações embasam-se no conceito da ecoequidade.

Além do mais, diversos elementos são necessários para desenvolver estruturas de sustentabilidade corporativa, pois cada definição oferece visões específicas. Legitimar as atividades corporativas, tanto dentro de uma empresa quanto na sociedade, é fundamental para os negócios e requer um comportamento corporativo responsável e ético (BAUMGARTNER, 2014). Martínez-Jurado e Moyano-Fuentes (2014) concluem que as crescentes pressões, das partes interessadas, exercem influência nas decisões sobre quais estratégias a empresa adotará, a fim de gerenciar seus negócios de forma responsável e ciente dos impactos que suas atividades representam na sociedade, e, portanto, aumentando sua responsabilidade social corporativa.

Diante do exposto, entende-se que as práticas de sustentabilidade demandam ações que possam ser reproduzidas e que possibilitem a interação com os fenômenos organizacionais. Assim, o fato de a sustentabilidade ser um estado alcançado por ações, as práticas de sustentabilidade organizacional envolvem aspectos relacionados às relações humanas e diversos entes da sociedade e do meio ambiente, portanto são orientadas por uma série de valores. Estes valores não financeiros representam um grande desafio aos modelos de negócios atuais, pois as necessidades impostas pelo mundo corporativo moderno têm obrigado as empresas a pensarem em alternativas para uma gestão cada vez mais eficiente.

Além disso, como os valores organizacionais subjazem os modelos de gestão, as empresas são vistas como um ente que atua em função de regras, procedimentos, crenças e valores predominantes em determinado contexto. Nesse sentido, a fim de compreender como as organizações associam as práticas de sustentabilidade ao seu modelo de negócios, a seguir discorre-se sobre como as organizações podem situar o modelo da TI Verde dentro do framework teórico da Sustentabilidade Corporativa. 


\section{MODELO PROPOSITIVO DA RELAÇÃO DA TI VERDE E SUSTENTABILIDADE EMPRESARIAL}

O modelo foi norteado pelo conceito da Triple Bottom Line (ELKINGTON; BURKE, 1987), em virtude de este fundamentar-se na teoria das partes interessadas, exigindo que as empresas meçam seu desempenho em relação às comunidades locais e governos e não apenas com as partes interessadas com as quais possuem relacionamentos diretos e transacionais, como funcionários, clientes e fornecedores.

Além disto, no atual cenário de economia de recursos faz-se mister a conscientização dos empresários quanto à necessidade de atuação de acordo com uma responsabilidade social que se concretiza no respeito aos direitos humanos, na melhoria da qualidade de vida da comunidade, da sociedade e na preservação do meio ambiente. Assim, entende-se que, por pensar nos recursos para as gerações futuras, torna-se imprescindível que as análises quanto ás práticas de TI Verde sejam embasadas em termos econômicos, ambientais e sociais, as mesmas perspectivas cunhadas no modelo da Triple Bottom Line. O Quadro 1 apresenta os construtos e as variáveis que foram delimitadas por meio da pesquisa bibliográfica, para cada uma das perspectivas de análise que serviram de base para a construção do modelo.

Quadro 1 - Perspectivas e constructos da TI Verde

(continua)

\begin{tabular}{|c|c|c|c|}
\hline Perspectivas & Construtos & Variáveis & Referencial \\
\hline \multirow{13}{*}{ 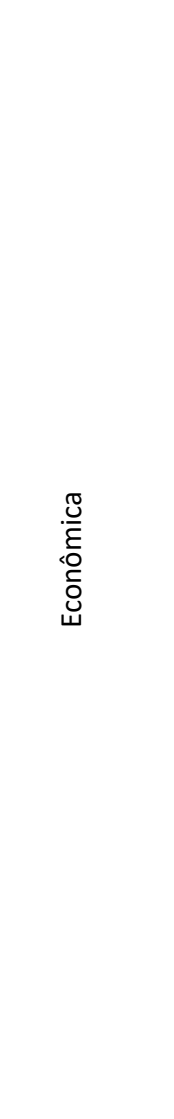 } & \multirow{4}{*}{$\begin{array}{l}\frac{0}{0} \\
\frac{\pi}{0} \\
.0 \\
\frac{0}{0} \\
\frac{\pi}{3} \\
J\end{array}$} & $\begin{array}{l}\text { O investimento em tecnologias limpas traz benefícios } \\
\text { econômicos. }\end{array}$ & $\begin{array}{l}\text { Molla et al., 2009; Kuo; } \\
\text { Dick, } 2009\end{array}$ \\
\hline & & $\begin{array}{l}\begin{array}{l}\text { Utiliza incentivo fiscal oferecido por órgãos } \\
\text { governamentais. }\end{array} \\
\end{array}$ & Chen et al., 2011. \\
\hline & & Utiliza software de gerenciamento de energia. & $\begin{array}{l}\text { Mellville, 2010; Uddin; } \\
\text { Rahman, } 2012 .\end{array}$ \\
\hline & & $\begin{array}{l}\text { Investe em tecnologias verdes, a fim de melhorar a } \\
\text { eficiência de recursos em TI. }\end{array}$ & $\begin{array}{l}\text { Chen et al., 2011; } \\
\text { Baumgartner, } 2014 .\end{array}$ \\
\hline & & $\begin{array}{l}\text { Redução de custos por meio da implementação de } \\
\text { iniciativas sustentáveis em TI. }\end{array}$ & $\begin{array}{l}\text { Molla et al., 2009; Watson; } \\
\text { Bourdreau; Chen, } 2010 .\end{array}$ \\
\hline & & A preocupação ambiental aumenta os custos. & Hart; Milstein, 2003 \\
\hline & & $\begin{array}{l}\text { Controlam-se os custos com manutenção de } \\
\text { equipamentos. }\end{array}$ & Unhelkar, 2011 \\
\hline & 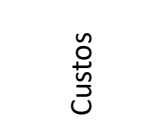 & $\begin{array}{l}\text { O gerenciamento inteligente da tecnologia é uma } \\
\text { alternativa para reduzir os custos operacionais do } \\
\text { negócio. }\end{array}$ & $\begin{array}{l}\text { Melville, 2010; Watson; } \\
\text { Bourdreau; Chen, 2010; } \\
\text { Bose; Luo, } 2011\end{array}$ \\
\hline & & $\begin{array}{l}\text { A fim de evitar desperdícios, os equipamentos de TI } \\
\text { possuem autonomia para aumentar sua eficiência } \\
\text { energética. }\end{array}$ & Unhelkar, 2011 \\
\hline & & $\begin{array}{l}\text { Os desperdícios podem ser transformados em } \\
\text { economia, por meio de práticas ecologicamente } \\
\text { corretas com relação ao uso da tecnologia. }\end{array}$ & $\begin{array}{l}\text { Mithas; Khuntia; Roy, } \\
\text { 2010; Molla; Abareshi, } \\
2011\end{array}$ \\
\hline & 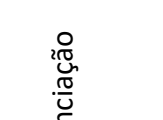 & $\begin{array}{l}\text { As iniciativas de cunho ambiental resultam em benefícios } \\
\text { à imagem da organização perante a sociedade. }\end{array}$ & $\begin{array}{l}\text { Sheth; Sethia; Srinivas, } \\
\text { 2011; Crittenden et al., } \\
\text { 2011; Hult, } 2011\end{array}$ \\
\hline & $\frac{\complement}{2}$ & Pode-se diferenciar no mercado por meio da TI Verde. & Cai; Chen; Bose, 2013 \\
\hline & $\stackrel{\frac{\omega}{0}}{0}$ & $\begin{array}{l}\text { A TI Verde aumenta a participação no mercado e } \\
\text { possibilita entrar em novos e mais lucrativos mercados. }\end{array}$ & Cai; Chen; Bose, 2013 \\
\hline
\end{tabular}




\begin{tabular}{|c|c|c|c|}
\hline \multirow{15}{*}{ 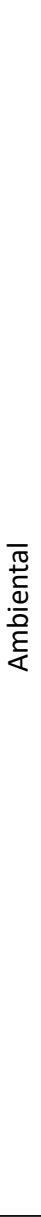 } & \multirow{2}{*}{ 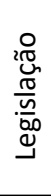 } & $\begin{array}{l}\text { Utiliza-se a regulamentação ambiental como } \\
\text { oportunidade para inovar. }\end{array}$ & Dangelico; Pujari, 2010 \\
\hline & & $\begin{array}{l}\text { A adoção de práticas atreladas à sustentabilidade } \\
\text { ambiental é motivada pela legislação. }\end{array}$ & $\begin{array}{l}\text { Molla, 2008: Bose; Luo, } \\
2011 ; \text { Aguinis; Glavas, } \\
2012\end{array}$ \\
\hline & \multirow{7}{*}{ 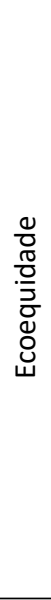 } & $\begin{array}{l}\text { A empresa se preocupa com o impacto ambiental } \\
\text { dos sistemas e os equipamentos de tecnologias de } \\
\text { informação. }\end{array}$ & Molla, 2008 \\
\hline & & $\begin{array}{l}\text { Política de utilização sustentável de recursos, como } \\
\text { diminuição de impressões. }\end{array}$ & Dick, Burns, 2011 \\
\hline & & $\begin{array}{l}\text { Busca-se reduzir os impactos ambientais que as } \\
\text { atividades de TI causam no meio ambiente. }\end{array}$ & Corbett, 2010 \\
\hline & & $\begin{array}{l}\text { A TI Verde pode reduzir a pegada de carbono da } \\
\text { empresa. }\end{array}$ & Unhelkar, 2011 \\
\hline & & $\begin{array}{l}\text { Os equipamentos eletrônicos obsoletos são coletados } \\
\text { por empresas especializadas. }\end{array}$ & Corbett, 2010 \\
\hline & & $\begin{array}{l}\text { Os equipamentos possuem um ciclo de vida, com } \\
\text { relação ao seu uso, estipulado pela empresa. }\end{array}$ & Elliot, 2007 \\
\hline & & $\begin{array}{l}\text { A empresa preocupa-se com os recursos das futuras } \\
\text { gerações. }\end{array}$ & Pollack, 2008 \\
\hline & \multirow{6}{*}{ 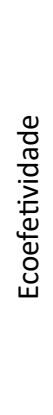 } & $\begin{array}{l}\text { As iniciativas que objetivam tornar as atividades de TI } \\
\text { sustentáveis são pontuais. }\end{array}$ & Chen et al., 2011 \\
\hline & & $\begin{array}{l}\text { Realiza-se a logística reversa dos equipamentos de TI, } \\
\text { devolvendo-os para o fornecedor após o uso. }\end{array}$ & Mont, 2002 \\
\hline & & $\begin{array}{l}\text { Prefere-se adquirir equipamentos que tenham selo } \\
\text { verde. }\end{array}$ & Corbett, 2010 \\
\hline & & A empresa possui alguma certificação ambiental. & Hubbard, 2009 \\
\hline & & Incentiva-se a reciclagem de equipamentos eletrônicos. & Corbett, 2010 \\
\hline & & $\begin{array}{l}\text { Realiza-se avaliação dos fornecedores de suprimentos, } \\
\text { do ponto de vista ambiental. }\end{array}$ & Hubbard, 2009 \\
\hline \multirow{6}{*}{$\frac{\bar{\pi}}{\overline{\mathrm{O}}}$} & \multirow{3}{*}{$\begin{array}{l}\frac{\mathscr{O}}{0} \\
\frac{\pi}{0} \\
\frac{0}{5} \\
\frac{\varepsilon}{0}\end{array}$} & $\begin{array}{l}\text { Os computadores que estão desatualizados para } \\
\text { as atividades da empresa, mas que ainda possuem } \\
\text { utilidade, são doados para filantropia. }\end{array}$ & Molla, 2008 \\
\hline & & $\begin{array}{l}\text { A adoção de práticas atreladas à sustentabilidade } \\
\text { ambiental é motivada pela pressão da sociedade. }\end{array}$ & Kuo; Dick, 2010 \\
\hline & & $\begin{array}{l}\text { Oportuniza-se para colaboradores e a comunidade } \\
\text { a coleta de lixo eletrônico, para descartá-lo de forma } \\
\text { adequada e segura. }\end{array}$ & Molla, 2009 \\
\hline & \multirow{3}{*}{ 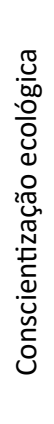 } & $\begin{array}{l}\text { São oferecidas palestras instrutivas para colaboradores } \\
\text { e comunidade, a fim de orientá-los quanto ao uso } \\
\text { consciente da tecnologia. }\end{array}$ & Fuchs, 2008 \\
\hline & & $\begin{array}{l}\text { Movimentos de conscientização dos funcionários } \\
\text { quanto à importância de utilizar os recursos, como } \\
\text { energia e papel, de forma consciente. }\end{array}$ & $\begin{array}{l}\text { Sarkar; Young, 2009; Dick; } \\
\text { Burns, 2011; Baumgartner, } \\
2014\end{array}$ \\
\hline & & $\begin{array}{l}\text { Devido às práticas de conscientização adotadas pela } \\
\text { empresa, os colaboradores tendem a adotar diferentes } \\
\text { posturas quanto ao uso da tecnologia em suas } \\
\text { residências e incentivam amigos e familiares a fazerem } \\
\text { o mesmo. }\end{array}$ & $\begin{array}{l}\text { Mishra; Akman; Mishra, } \\
2014 \text {; Koo; Chung; Nam, } \\
2015\end{array}$ \\
\hline
\end{tabular}

Fonte: Elaborado pelos autores.

A partir das bases teóricas apresentadas, verificou-se que a necessidade de maior eficiência da TI (POLLACK, 2008; MOLLA et al., 2009; MELVILLE, 2010; UNHELKAR, 2011; LOOS et al., 2011) e a busca de economia de custos tangíveis das operações de TI (MOLLA, 2009; POLLACK, 
2008; MOLLA et al., 2009; MITHAS; KHUNTIA; ROY, 2010; WATSON; BOUDREAU; CHEN, 2010; MELVILLE, 2010; BOSE; LUO, 2011; UNHELKAR, 2011; KOO; CHUNG; NAM, 2015) são os principais fatores motivacionais para a adoção da TI Verde. Mesmo, contudo, que o principal fator motivacional sejam os fatores econômicos, constatou-se que a prática de TI Verde reflete positivamente no meio ambiente e na sociedade.

Logo, entende-se que para situar o modelo da TI Verde no contexto teórico da Sustentabilidade Corporativa, deve-se considerá-lo um processo cíclico, pois trata-se de uma atividade contínua no ambiente organizacional, que resulta da combinação de motivos, tanto de natureza objetiva e técnica como subjetiva e comportamental. Assim, fundamentando-se nas perspectivas e construtos apresentados no Quadro 1, bem como nas bases conceituais elencados, a Figura 1 ilustra o modelo proposto, denominado ciclo estratégico da TI Verde.

Figura 1 - Ciclo estratégico da TI Verde

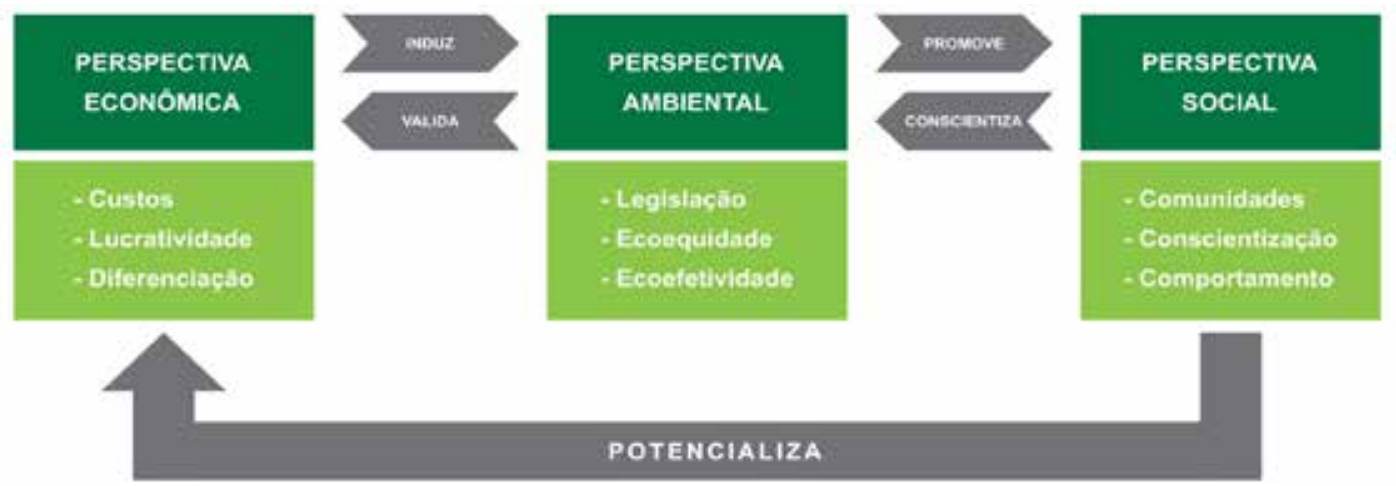

Fonte: Elaborado pelos autores.

Para as empresas, o pensamento econômico engloba, primordialmente, a redução de custos, aumento dos lucros e na participação de novos mercados. O gerenciamento inteligente da tecnologia é uma alternativa para reduzir os custos operacionais do negócio (MELVILLE, 2010; WATSON; BOUDREAU; CHEN, 2010; BOSE; LUO, 2011) e pode ser feito utilizando-se softwares de gerenciamento de energia no parque de computadores, de forma que a infraestrutura de TI tenha autonomia para aumentar sua eficiência energética, a fim de evitar desperdícios (UDDIN; RAHMAN, 2012). Os benefícios são mensurados, não apenas na diminuição do consumo de energia, mas na redução dos gastos com depreciação e manutenção (UNHELKAR, 2011).

Visto que os benefícios ambientais não geram motivações suficientes para a intituição da TI Verde (MOLLA; ABARESHI, 2011), constatou-se que a perspectiva ambiental tende a ser inserida devido às restrições de recursos (BOSE; LUO, 2011) e legislações (MOLLA, 2009, SARKAR; YOUNG, 2009; MOLLA; ABARESHI, 2011).Quanto aos critérios de produção, operação e comercialização, que visam, principalmente, à redução do impacto ambiental da atividade (MOLLA et al., 2009) e responsabilidade quanto aos resíduos eletrônicos gerados (CORBETT, 2010). Em contrapartida, uma visão mais estratégica, do ponto de vista da sustentabilidade das Tecnologias de Informação e Comunicação, pode resultar em oportunidades de negócios.

Nesse sentido, as empresas podem aumentar sua diferenciação no mercado por meio de proventos intangíveis associados à TI Verde (CRITTENDEN et al., 2011). Isso se reflete em ações de ecoequidade e ecoefetividade, que possibilitam atuar de forma proativa no que respeita aos aspectos de responsabilidade socioambiental com as futuras gerações, não se limitando apenas 
em agir de forma ambientalmente correta para atender às legislações ambientais ou em prol dos benefícios financeiros diretos. Logo, a TI pode direcionar as empresas à sustentabilidade ecológica, ao inserir inovações que mudam a lógica de comercialização ou uso de produtos ou serviços (CHEN et al. (2011).

Além disto, ao adotarem uma postura proativa, com relação à sustentabilidade ambiental e aproveitar as variáveis ecológicas como fator estratégico no desenvolvimento de inovações com maior grau de radicalismo, as empresas entram em novos e lucrativos mercados. Entende-se que, no aspecto ambiental, as empresas têm a oportunidade de repensar seu modelo de negócio, uma vez que podem utilizar as regulamentações ambientais como oportunidade para inovar nos produtos e processos, a fim de desenvolver soluções e produtos que impactem positivamente no ambiente no qual estão inseridas, além de agregar valor para sua marca, produtos e serviços.

Ademais, a legitimação das atividades corporativas, tanto internamente quanto com a sociedade, é fundamental para os negócios e requer uma atuação responsável e ética (BAUMGARTNER, 2014). Assim, acredita-se que o motivador ético se relaciona com a busca de práticas comerciais socialmente responsáveis e a boa cidadania corporativa. Nesta perspectiva, Molla (2008) sinaliza que na medida que o movimento verde permeia todos os aspectos do quotidiano corporativo, as empresas estão buscando o reconhecimento social como entidades interessadas na comunidade global e local.

Seguindo este encadeamento temático, contata-se que a perspectiva social da TI Verde aumenta a credibilidade da companhia perante o mercado, visto que o desempenho social retrata o impacto que uma empresa e seus fornecedores geram nas comunidades em que operam (HUBBARD, 2009). Ademais, novas expectativas sociais tornam imperativo para as empresas entender como integrar questões de sustentabilidade às estratégias de negócios, uma vez que, a relação destas estratégias resulta na diferenciação e aumento de vantagem competitiva no mercado, e, consequentemente, agregação de valor (DANGELICO; PUJARI, 2010).

Outro impacto positivo, advindo da perspectiva social da TI Verde, diz respeito à conscientização e comportamento dos indivíduos com relação ao uso das tecnologias. A literatura demonstrou que, devido às práticas de conscientização quanto ao uso consciente da tecnologia, os colaboradores tendem a adotar o mesmo comportamento e conscientização na sua vida pessoal, estendendo tais práticas e conhecimentos entre amigos e familiares, de forma a contribuir para a redução dos impactos ambientais (BAUMGARTNER, 2014; MISHRA; AKMAN; MISHRA, 2014). Sumariamente, pode-se inferir que a TI Verde aumenta o nível de conscientização ambiental e muda o comportamento dos indivíduos.

Com base nestas considerações, percebe-se que a perspectiva econômica da TI Verde induz à adoção da perspectiva ambiental. Conforme as empresas integram as práticas ambientais às econômicas, por meio das regulamentações ambientais e das práticas de ecoequidade (BAUMGARTNER, 2014) e ecoefetividade (MELVILLE, 2010; CHEN et al.; 2011), valida-se a perspectiva econômica, por meio das oportunidades para minimização de riscos, preservação de receitas e reputação, maior eficiência no uso de recursos, retorno sobre o investimento, aumento de vendas, desenvolvimento de novos mercados e melhor imagem corporativa (HUBBARD, 2009; DANGELICO; PUJARI, 2010; HULT, 2011; SHETH; SETHIA; SRINIVAS, 2011; CRITTENDEN et al., 2011; CAl; CHEN; BOSE, 2013). 
No que diz respeito à questão social, esta é inserida no ambiente corporativo como consequência das práticas ambientais e potencializa as variáveis de lucratividade e diferenciação no mercado. Ademais, as práticas de TI Verde contribuem para a constituição da identidade organizacional, possibilitando maior transparência de seus valores, ações e padrões, estabelecidos e corroborados pelos atores sociais, ao mesmo tempo que são reconhecidos pela sociedade, favorecendo a legitimidade da organização. Logo, a adoção destas práticas permite o controle de riscos e oportunidades de negócios e possibilita que não apenas a empresa, mas também sua cadeia de valor, utilizem estratégias e ferramentas rumo à gestão socialmente responsável.

\section{CONSIDERAÇÕES FINAIS}

Neste artigo, baseado em uma revisão sistemática de literatura sobre as temáticas de Sustentabilidade Corporativa e TI Verde, foi promovido o diálogo entre os autores que subsidiaram a concepção de um modelo que situa a TI Verde no contexto teórico da Sustentabilidade Corporativa. Em termos estruturais, o modelo foi norteado pelo conceito da Triple Bottom Line, em virtude de este ser considerado um modelo de gestão de negócios que visa ao lucro para os acionistas, envolvendo, a um só tempo, o desenvolvimento econômico, a promoção social e a proteção dos recursos naturais do planeta, respectivamente resumidas nas perspectivas econômicas, social e ambiental.

Ainda que esta pesquisa tenha atingido o objetivo proposto e que o rigor metodológico tenha sido perseguido, não se pode desconhecer a existência de limitações. A primeira delas diz respeito ao viés de interpretação dos autores durante o processo de análise. Destaca-se ainda a limitação de ordem operacional, visto que, ao se realizar a busca dos artigos em portais de pesquisas específicos, os resultados propostos são pertinentes às bases de dados consultadas. Cumpre destacar que, ao utilizar o número de citações como critérios de seleção dos artigos para análise, estudos posteriores não encontrarão o mesmo comportamento do ranking apresentado nesta investigação teórica.

Ressalta-se que os resultados obtidos indicam possibilidades para a continuidade da linha da pesquisa. Nesse sentido, cumpre destacar que a necessidade de maior eficiência energética e, consequentemente, economia de custos tangíveis nas operações de $\mathrm{Tl}$, são os principais fatores para adoção da TI Verde. Logo, em termos de motivação, o valor econômico contínuo no longo prazo influencia o engajamento das práticas de TI Verde no âmbito organizacional. Embora diversos estudos tenham sido realizados, em termos de conservação de energia (CHUNG, 2017; FANG et al., 2015; UDDIN; RAHMAN, 2012) e redução de custos (JONGSAGUAN; GHONEIM, 2017; KURKALOVA; CARTER, 2017) advindos da instituição das práticas de TI Verde, estes restringem-se a modelos teóricos.

Com base, portanto, nos resultados deste estudo, foi possível constatar que pesquisas empíricas que mensuram a redução de custos advinda das iniciativas de TI Verde, o desempenho operacional ou o valor de mercado das empresas são escassas na literatura atual. Nesta perspectiva, Bose e Luo (2011) reconhecem que é difícil obter este tipo de informação por meio das pesquisas de campo, devido à dificuldade em realizar a coleta de dados junto as empresas. Desta forma, sugere-se que as universidades e órgãos públicos trabalhem em conjunto, no sentido de mobilizar as empresas a disponibilizarem suas informações, de modo confidencial, para a produção de novos conhecimentos. O envolvimento do governo também pode proporcionar 
mais estudos no âmbito da sociedade, uma vez que as investigações sobre os benefícios sociais advindos da adoção da TI Verde são raras.

Como reflexão final, destaca-se o potencial que as empresas apresentam em influenciar o contexto em que atuam, visto que devido às práticas de conscientização quanto ao uso consciente dos recursos de tecnologia, os indivíduos tendem a adotar o mesmo comportamento e conscientização na sua vida pessoal. Nesse sentido, acredita-se que o envolvimento dos indivíduos em ações e atividades que dizem respeito à sustentabilidade ambiental contribuem para melhorar a qualidade de vida e preservar os recursos naturais.

\section{REFERÊNCIAS}

AGUINIS, Herman; GLAVAS, Ante. What we know and don't know about corporate social responsibility: A review and research agenda. Journal of Management, v. 38, n. 4, p. 932-968, 2012.

ASADI, Shahla; HUSSIN, Ab Razak Che; DAHLAN, Halina Mohamed. Organizational research in the field of Green IT: A systematic literature review from 2007 to 2016 . Telematics and Informatics, v. 34, n. 7, p. 1.191-1.249, 2017.

BAUMGARTNER, Rupert J.; EBNER, Daniela. Corporate sustainability strategies: sustainability profiles and maturity levels. Sustainable Development, v. 18, n. 2, p. 76-89, 2010.

BAUMGARTNER, Rupert J. Managing corporate sustainability and CSR: A conceptual framework combining values, strategies and instruments contributing to sustainable development. Corporate Social Responsibility and Environmental Management, v. 21, n. 5, p. 258-271, 2014.

BOSE, Ranjit; LUO, Xin. Integrative framework for assessing firms' potential to undertake Green IT initiatives via virtualization - A theoretical perspective. The Journal of Strategic Information Systems, v. 20, p. 38-54, 2011.

BUTLER, Tom. Compliance with institutional imperatives on environmental sustainability: Building theory on the role of Green IS. The Journal of Strategic Information Systems, v. 20, p. 6-26, 2011.

CAI, Shun; CHEN, Xi; BOSE, Indranil. Exploring the role of IT for environmental sustainability in China: An empirical analysis. International Journal of Production Economics, v. 146, n. 2, p. 491-500, 2013.

CHEN, Adela J.; BOUDREAU, Marie-Claude; WATSON, Richard T. Information systems and ecological sustainability. Journal of Systems and Information Technology, Sustainability and Information Systems, v. 10, n. 3, p. 186-201, 2008.

CHEN, Yu-Shan; CHANG, Ching-Hsun; WU, Feng-Shang. Origins of green innovations: the differences between proactive and reactive green innovations. Management Decision, v. 50, n. 3, p. 368-398, 2012.

CHEN, Adela J. et al. An Institutional Perspective on the Adoption of Green IS \& IT. Australasian Journal of Information Systems, v. 17, p. 5-27, 2011.

CHUNG, Yao-Liang. Energy-Saving Transmission for Green Macrocell-Small Cell Systems: A System-Level Perspective. IEEE Systems Journal, v. 11, n. 2, p. 706-716, 2017.

CORBETT, Jacqueline. Unearthing the Value of Green It. INTERNATIONAL CONFERENCE ON INFORMATION SYSTEMS - ICIS, 31., 2010. Proceedings [...]. Saint Louis, Missouri, USA, 2010.

CRITTENDEN, Victoria L. et al. Market-oriented sustainability: a conceptual framework and propositions. Journal of the Academy of Marketing Science, v. 39, n. 1, p. 71-85, 2011.

DANGELICO, Rosa Maria; PUJARI, Devashish. Mainstreaming green product innovation: Why and how companies integrate environmental sustainability. Journal of Business Ethics, v. 95, n. 3, p. 471-486, 2010. DENG, Qi; JI, Shaobo; WANG, Yun. Green IT practice disclosure. Journal of Information, Communication and Ethics in Society, v. 15, p. 145-64, 2017.

DICK, Geoffrey N.; BURNS, Max. Green IT in small business: an exploratory study. In: SOUTHERN ASSOCIATION OF INFORMATION SYSTEM CONFERENCE, 2011. Atlanta. Proceedings [...]. Atlanta, GA, USA, Mar. 25th-26th 2011.

DYLLICK, Thomas; HOCKERTS, Kai. Beyond the business case for corporate sustainability. Business Strategy and the Environment, v. 11, n. 2, p. 130-141, 2002.

EISENHARDT, Kathleen M. Building theories from case study research. Academy of Management Review, v. 14, n. 4, p. 532-550, 1989. 
ELLIOT, Steve. Environmentally sustainable ICT: a critical topic for IS research. PACIFIC ASIA CONFERENCE INFORMATION SYSTEMS (PACIS), 11., 2007. Auckland. Proceedings [...]. Auckland, New Zealand, 2007.

ELKINGTON, John; BURKE, Tom. The Green Capitalists: How Industry Can Make Money - and Protect the Environment. London: Victor Gollancz, 1987. 258 p.

FANG, Chao et al. A Survey of Green Information-Centric Networking: Research Issues and Challenges. IEEE Communications Surveys \& Tutorials, v. 17, n. 3, p. 1.455-1.472, 2015.

FINK, Arlete G. Conducting Research Literature Reviews: From the Internet to Paper. SAGE Publications. 4. ed. 2014. 280 p.

FUCHS, Christian. The implications of new information and communication technologies for sustainability. Environment, Development and Sustainability, v. 10, n. 3, p. 291-309, 2008.

GARCÍA-BERNÁ, José A. et al. Green IT and sustainable technology development: Bibliometric overview. Sustainable Development, v. 27, n. 4, p. 613-636, 2019.

HART, Stuart L.; MILSTEIN, Mark B. Creating sustainable value. The Academy of Management Executive, v. 17 , n. 2, p. 56-67, 2003.

HUBBARD, Graham. Measuring organizational performance: beyond the triple bottom line. Business strategy and the environment, v. 18, n. 3, p. 177-191, 2009.

HULT, G. Tomas M. Market-focused sustainability: market orientation plus! Journal of the Academy of Marketing Science, v. 39, n.1, p. 1-6, 2011.

JENKIN, Tracy A.; WEBSTER, Jane; MCSHANE, Lindsay. An agenda for "Green" information technology and systems research. Information and Organization, v. 21, n. 1, p. 17-40, 2011.

JONGSAGUAN, Salakjit; GHONEIM, Ahmad. Green IT/IS investments evaluation within the aviation industry: A focus on indirect cost management. Journal of Enterprise Information Management, v. 30, n. 2, p. 206-225, 2017.

KITCHENHAM, Barbara et al. Systematic literature reviews in software engineering: a tertiary study. Information \& Software Technology, v. 52, n. 8, p. 792-805, 2010.

KOO, Chulmo; CHUNG, Namho; NAM, Kichan. Assessing the impact of intrinsic and extrinsic motivators on smart green IT device use: Reference group perspectives. International Journal of Information Management, v. 35, n. 1, p. 64-79, 2015.

KUO, Ben; DICK, Geoffrey. The greening of organisational IT: what makes a difference? Australasian Journal of Information Systems, v. 16, n. 2, 2010.

KURKALOVA, Lyubov A.; CARTER, Lemuria. Sustainable production: Using simulation modeling to identify the benefits of green information systems. Decision Support Systems, v. 96, p. 83-91, 2017.

LOOS, Pete et al. Green IT: A Matter of Business and Information Systems Engineering? Business \& Information Systems Engineering, v. 3, n. 4, p. 245-252, 2011.

MALHOTRA, Arvind; MELVILLE, Nigel P.; WATSON, Richard T. Spurring impactful research on information systems for environmental sustainability. MIS Quarterly, v. 37, n. 4, p. 1.265-1.274, 2013.

MARTÍNEZ-JURADO, Pedro José; MOYANO-FUENTES, José. Lean management, supply chain management and sustainability: a literature review. Journal of Cleaner Production, v. 85, p. 134-150, 2014.

MELVILLE, Nigel P. Information Systems Innovation for Environmental Sustainability. MIS Quarterly, v. 34, p. 1-21, 2010.

MISHRA, Deepti; AKMAN, Ibrahim; MISHRA, Alok. Theory of reasoned action application for green information technology acceptance. Computers in Human Behavior, v. 36, p. 29-40, 2014.

MITHAS, Sunil; KHUNTIA, Jiban; ROY, Prasanto K. Green Information Technology, Energy Efficiency, and Profits: Evidence from an Emerging Economy. In: ICIS, 2010. Proceedings [...]. 2010. p. 11. Disponível em: https://aisel.aisnet.org/icis2010_submissions/11

MOLLA, Alemayehu et al. An International Comparison of Green IT Diffusion. International Journal of e-Business Management, v. 3, n. 2, p. 3-23, 2009.

MOLLA, Alemayehu; ABARESHI, Ahmad. Green It Adoption: A Motivational Perspective. PACIFIC ASIA CONFERENCE ON INFORMATION SYSTEMS (PACIS), 2011. Proceedings [...]. 2011. p. 137.

MOLLA, Alemayehu; ABARESHI, Ahmad. Organizational Green Motivations for Information Technology: Empirical Study. The Journal of Computer Information Systems, v. 52, n. 3, p. 92-102, 2012.

MONT, Oksana K. Clarifying the concept of product-service system. Journal of cleaner production, v. 10, n. 3, p. 237-245, 2002.

ONU. Organização das Nações Unidades. Our Future Common. 1987. Disponível em: http://www.un-documents. net/wced-ocf.htm. Acesso em: 4 abr. 2018. 
POLLACK, Thomas A. Green and Sustainable Information Technology: A foundation for Students. ASCUE, 2008. Proceedings [...]. Pittsburgh: Duquesne University, 2008.

RIDLEY, Diana. The literature review: A step-by-step guide for students. 2. ed. Los Angeles; London; Nova Delhi; Singapura; Washington DC: Sage Study Skills, 2012.

SARKAR, Pradipta; YOUNG, Leslie. Managerial attitudes towards Green IT: an explorative study of policy drivers. PACIFIC ASIA CONFERENCE ON INFORMATION SYSTEMS - (PACIS), 2009. Hyderabad. Proceedings [...]. Hyderabad, India, 2009. p. 95.

SHETH, Jagdish N.; SETHIA, Nirmal K.; SRINIVAS, Shanthi. Mindful consumption: a customer-centric approach to sustainability. Journal of the Academy of Marketing Science, v. 39, n. 1, p. 21-39, 2011.

SIMS, Joanne L.; MCGHEE, Charles NJ. Citation analysis and journal impact factors in ophthalmology and vision science journals. Clinical \& Experimental Ophthalmology, v. 31, n. 1, p. 14-22, 2003.

UDDIN, Mueen; RAHMAN, Azizah Abdul. Energy efficiency and low carbon enabler green IT framework for data centers considering green metrics. Renewable and Sustainable Energy Reviews, v. 16, n. 6, p. 4078-4094, 2012.

UNHELKAR, Bhuvan. Green IT Strategies and Applications: Using Environmental Intelligence. Boca Raton, Flórida: CRC Press, 2011.

WATSON, Richard T.; BOUDREAU, Marie-Claude; CHEN, Adela J. Information systems and environmentally sustainable development: energy informatics and new directions for the IS community. MIS Quarterly, v. 34, n. 1, p. 23-38, mar., 2010.

WEBSTER, Jane; WATSON, Richard T Analyzing the past to prepare for the future: Writing a literature review. MIS Quarterly, v. 26, n. 2, p. 13-23, 2002.

WOLFSWINKEL, Joost. F.; FURTMUELLER, Elfi.; WILDEROM, Celeste P. M. Using grounded theory as a method for rigorously reviewing literature. European Journal of Information Systems, v. 22 n. 1, p. 45-55, 2013. 